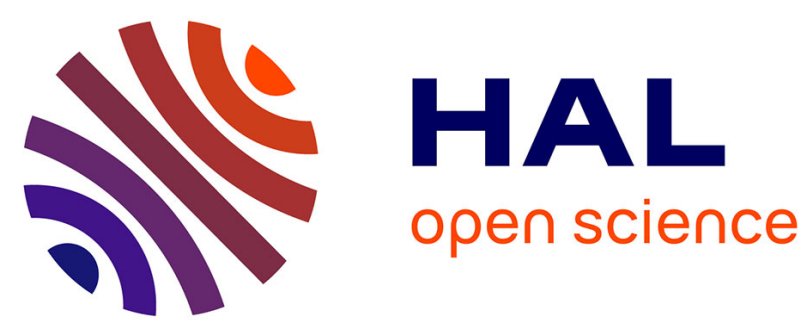

\title{
Macro-atom versus many-electron effects in ultrafast ionization of $\mathrm{C} 60$
}

\author{
Y. Huismans, E. Cormier, C. Cauchy, Paul-Antoine Hervieux, G. Gademann,
} A. Gijsbertsen, O. Ghafur, P. Johnsson, P. Logman, T. Barillot, et al.

\section{- To cite this version:}

Y. Huismans, E. Cormier, C. Cauchy, Paul-Antoine Hervieux, G. Gademann, et al.. Macro-atom versus many-electron effects in ultrafast ionization of C 60. Physical Review A : Atomic, molecular, and optical physics [1990-2015], 2013, 88 (1), pp.013201. 10.1103/PhysRevA.88.013201 . hal-03060530

\section{HAL Id: hal-03060530 \\ https://hal.science/hal-03060530}

Submitted on 14 Dec 2020

HAL is a multi-disciplinary open access archive for the deposit and dissemination of scientific research documents, whether they are published or not. The documents may come from teaching and research institutions in France or abroad, or from public or private research centers.
L'archive ouverte pluridisciplinaire HAL, est destinée au dépôt et à la diffusion de documents scientifiques de niveau recherche, publiés ou non, émanant des établissements d'enseignement et de recherche français ou étrangers, des laboratoires publics ou privés. 


\title{
Macro-atom versus many-electron effects in ultrafast ionization of $\mathbf{C}_{60}$
}

\author{
Y. Huismans, ${ }^{1}$ E. Cormier ${ }^{2}$ C. Cauchy,${ }^{3}$ P.-A. Hervieux,${ }^{4}$ G. Gademann, ${ }^{1}$ A. Gijsbertsen, ${ }^{1}$ O. Ghafur, ${ }^{1}$ P. Johnsson, ${ }^{1}$ \\ P. Logman, ${ }^{1}$ T. Barillot, ${ }^{3}$ C. Bordas, ${ }^{3}$ F. Lépine, ${ }^{3, *}$ and M. J. J. Vrakking ${ }^{1,5}$ \\ ${ }^{1}$ FOM-Institute AMOLF, Science Park 104, 1098 XG Amsterdam, The Netherlands \\ ${ }^{2}$ Université Bordeaux, CNRS, CEA, Ctr Lasers Intenses \& Application CELIA, F-33405 Talence, France \\ ${ }^{3}$ Institut Lumière Matière, UMR5306, Université Lyon 1-CNRS, 69622 Villeurbanne cedex, France \\ ${ }^{4}$ Institut de Physique et Chimie des Matériaux de Strasbourg, CNRS and University of Strasbourg, \\ 23 rue du Loess - BP 43 - 67034 Strasbourg Cedex, France \\ ${ }^{5}$ Max-Born Institut, Max-Born Strasse 2A, 12489 Berlin, Germany
}

(Received 21 January 2013; published 3 July 2013)

\begin{abstract}
Ionization mechanisms of $\mathrm{C}_{60}$ molecules irradiated by a short intense 800 -nm laser pulse are studied. Angleresolved photoelectron spectra show above-threshold ionization (ATI) patterns with a low peak contrast and a remarkably smooth angular distribution. The results are interpreted by combining two theoretical models. A time-dependent Schrödinger equation (TDSE) calculation based on the $B$-spline method that explicitly takes into account the molecular potential mimics the single-active-electron response while a statistical model accounts for the many-electron effects. We show that the latter are responsible for the loss of contrast in the ATI peaks. The smooth angular distribution arises in the TDSE calculation as a result of the high angular momentum of the $\mathrm{C}_{60}$ ground electronic state and therefore is a manifestation of the atomic behavior of the molecule.
\end{abstract}

DOI: 10.1103/PhysRevA.88.013201 PACS number(s): 36.40.-c, 33.20.Xx, 33.80.Eh, 33.80.Wz

The interaction between an intense light pulse and a complex polyatomic molecule is an archetype of many-body problems where the light electric field interacts with the electronic degrees of freedom inducing different types of dynamics where both electrons and nuclei may play a role. Ionization is one of the possible responses and may, due to the various degrees of freedom involved, occur on different time scales. Recent progress in light sources offers the possibility to investigate this dynamics down to the attosecond time scale and to observe the electronic motions in real time [1]. This motivates current work on electronic processes in complex systems.

In atomic species, ionization by an intense laser field typically leads to above-threshold ionization (ATI), in which the atom absorbs more than the minimum number of photons required to reach the ionization threshold. Extensive work on atomic ATI, largely based on photoelectron spectroscopy, has been focused on the distinction between tunneling and multiphoton ionization [2], the role of resonances [3], and the importance of electron interferences [4]. This kind of information is directly encoded in the photoelectron angular distribution. The case of molecules is far less investigated, but in a recent paper, van der Zwan et al. [5] have shown the influence of molecular orbitals on angle-resolved ATI. Corkum and co-workers also revealed the importance of diffractive effects during the electron recollision process (in which the electron is ejected by tunnel ionization, accelerated by the light electric field, and rescattered on the ionic core) resulting from the structure of the molecule [6]. In the more general case of complex polyatomic species, it is unclear whether the photoelectron energy and angular distribution can provide insight into the ionization dynamics and whether such processes can be adequately described in the singleactive-electron (SAE) picture or require one to account for the multielectron dynamics. $\mathrm{C}_{60}$ can be considered as a

\footnotetext{
*franck.lepine@univ-lyon1.fr
}

perfect "laboratory" to investigate these questions because a large variety of dissociation and ionization processes have already been experimentally observed [7]. In a seminal article, Campbell et al. [8] observed the modification of the $\mathrm{C}_{60}$ photoelectron spectrum as a function of the laser pulse duration. These changes were attributed to the gradually increasing influence of electronic and nuclear degrees of freedom on the multiphoton excitation process when the pulse duration was varied from 25 fs to 5 ps. When using longer laser pulses, multiphoton excitation leads to an efficient delayed electron emission driven by vibrational autoionization mechanisms, as revealed by the observation of an isotropic electron angular distribution that emphasizes the stochastic nature of the emission process [9]. In the case of short (9 fs) laser pulses, measurements of the ionization yield have shown that the excitation is driven by single-active-electron processes [10]. However, a multi-active-electron (MAE) mechanism has been invoked to explain the observation of Rydberg excitation [11], and recent experiments [12] have shown the importance of recollision processes and doorway states (corresponding to specific states that are populated during the excitation mechanism and that subsequently ionize) when the light intensity reaches $10^{14} \mathrm{~W} / \mathrm{cm}^{2}$. This demonstrates that the role played by the many-electron nature of the $\mathrm{C}_{60}$ remains a central question in the understanding of the ionization of $\mathrm{C}_{60}[13,14]$.

In this paper, we present an experiment in which $\mathrm{C}_{60}$ was ionized by a short 30 -fs IR pulse, with intensities ranging from $10^{12}$ up to $10^{14} \mathrm{~W} / \mathrm{cm}^{2}$ where for intensities below $5 \times 10^{13} \mathrm{~W} / \mathrm{cm}^{2}$ multiphoton processes dominate, while recollision-induced ionization and field ionization (i.e., tunneling and/or over-the-barrier ionization) are unexpected. The full three-dimensional momentum distribution of the electron was recorded with a velocity map imaging spectrometer. The measurements disentangle both aspects of the atomiclike ionization process that can be modeled using TDSE calculations and contributions involving electron-electron interactions that can be described by a statistical model for finite-size systems. 

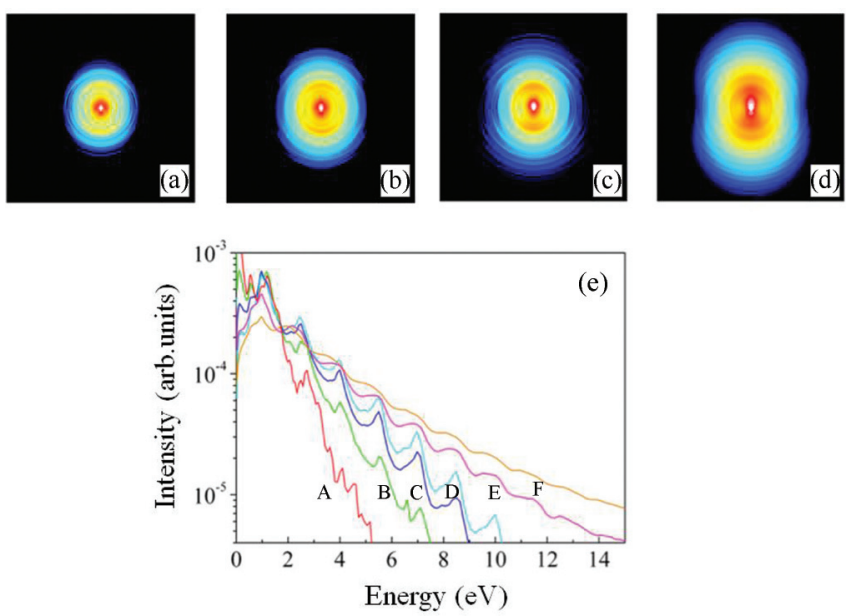

FIG. 1. (Color online) (a-d) Experimental photoelectron momentum distributions from the ionization of $\mathrm{C}_{60}$ with a $30-\mathrm{fs}, 800$-nm laser pulse. The four images shown were obtained for peak intensities of (a) $1.1 \times 10^{12} \mathrm{~W} / \mathrm{cm}^{2}$, (b) $9 \times 10^{12} \mathrm{~W} / \mathrm{cm}^{2}$, (c) $2.8 \times 10^{13} \mathrm{~W} / \mathrm{cm}^{2}$, and (d) $1.0 \times 10^{14} \mathrm{~W} / \mathrm{cm}^{2}$. A typical ATI pattern is observed. When the light intensity increases, the peak-to-valley contrast of the ATI peaks is reduced, as observed in the angle-integrated photoelectron spectra shown in (e). The angle-integrated photoelectron spectra are shown for intensities of (A) $1.1 \times 10^{12} \mathrm{~W} / \mathrm{cm}^{2}$, (B) $9 \times 10^{12} \mathrm{~W} / \mathrm{cm}^{2}$, (C) $2 \times 10^{13} \mathrm{~W} / \mathrm{cm}^{2}$, (D) $2.8 \times 10^{13} \mathrm{~W} / \mathrm{cm}^{2}$, (E) $6.7 \times 10^{13} \mathrm{~W} / \mathrm{cm}^{2}$, and $(\mathrm{F}) 1.0 \times 10^{14} \mathrm{~W} / \mathrm{cm}^{2}$.

Our experimental setup combined a molecular beam, a velocity map imaging spectrometer, and a focused, moderately intense femtosecond laser beam. The $\mathrm{C}_{60}$ molecular beam was produced with an oven operated at a temperature of $550{ }^{\circ} \mathrm{C}$. The molecular beam was collimated by a 1-mm skimmer and crossed the IR fs laser beam $(30 \mathrm{fs}, 800 \mathrm{~nm}, 4 \mathrm{kHz})$ that was focused with a $20-\mathrm{cm}$ focal lens. The two beams crossed on the axis of a velocity map imaging spectrometer designed according to the standard geometry proposed by Eppink and Parker [15]. Using this spectrometer, particles ejected with the same velocity vector are found on the same position of a position sensitive detector, which was made of a pair of 70-mm-diameter microchannel plates followed by a phosphor screen and a CCD camera.

Typical images obtained in our experiment for peak intensities of $10^{12}-10^{14} \mathrm{~W} / \mathrm{cm}^{2}$ are shown in Figs. 1(a)-1(d), along with a series of angle-integrated photoelectron kinetic energy distributions spanning peak intensities from $10^{12}$ to $10^{14} \mathrm{~W} / \mathrm{cm}^{2}$ [Fig. 1(e)]. In these electron momentum maps, concentric rings spaced by the energy of one IR photon are observed, which is the usual signature of ATI that corresponds to the absorption of additional photons by the electron. A smooth angular distribution is observed for every ATI ring, which becomes more peaked along the laser polarization axis when the ATI order increases. Usually, in atomic ATI, the photoelectron angular distribution is highly structured, due to the existence of Freeman resonances with high angular momentum and due to interferences between electron wave packets that are ionized at different times within a laser cycle [16]. For a complex system such as $\mathrm{C}_{60}$, one might expect the high electronic density of states to be responsible for the observed smooth angular distribution. However, in the following, we will present three-dimensional (3D) TDSE calculations adapted for $\mathrm{C}_{60}$ that show that the smooth angular distributions can very well be understood in terms of a purely atomic, single-active-electron effect. Besides the ATI structure, additional structures below $1 \mathrm{eV}$ are observed in the angle-integrated photoelectron spectra in Fig. 1(e). These structures were previously observed and interpreted as one-photon ionization out of high-lying Rydberg states that are populated by multiphoton nonadiabatic excitation [12].

In nonresonant ATI experiments with short laser pulses, peaks are expected at energies $E_{n}=n \omega_{\text {laser }}-V_{\text {ion }}-U_{p}$, where $\omega_{\text {laser }}$ is the laser frequency, $V_{\text {ion }}$ is the ionization potential, and $U_{p}=I_{\text {laser }} / 4 \omega_{\text {laser }}^{2}$ is the ponderomotive energy, with $I_{\text {laser }}$ the laser intensity. Consequently, with increasing laser intensity, the ATI peaks shift towards lower energy. Beside the shift, we also observe a progressive lowering of the ATI peak energies with intensity, as well as a disappearance of the ATI structure at intensities close to $10^{14} \mathrm{~W} / \mathrm{cm}^{2}$. This trend is partially explained by the role of focal volume averaging in which different intensities in the laser beam profile induce different energy shifts, but is mostly attributed to the onset of multiple ionization at higher intensities. Neither mechanism can explain the low ATI peak contrast observed at low intensities such as $2 \times 10^{13} \mathrm{~W} / \mathrm{cm}^{2}$. As pointed out by Campbell et al. [8], we will show that the loss of contrast in this intensity range is due to the coexistence of direct and indirect (thermoelectronic-like) emission.

The interaction of $\mathrm{C}_{60}$ with an ultra-intense laser field requires consideration of the following issues. First of all, the coupling between the molecule and the laser beam occurs through the dipole interaction. This can be modeled in the SAE approximation by a TDSE calculation. However, the many-electron structure of the molecule results in a collective behavior where energy is transferred to the electronic cloud with the consequence of smearing out the single-activeelectron aspects. This is modeled by applying statistical theory. Though negligible in our experiment, the coupling of the electrons to phonons, which leads to vibrational autoionization on longer time scales, is also taken into account. To account for the fact that the $\mathrm{C}_{60}$ molecules experience different laser intensities depending on their location in the laser beam, focal volume averaging is required in the modeling.

In the following, atomic units are used unless otherwise specified:

For each single molecule, the response to the incident laser field is calculated in the SAE approximation by solving the $3 \mathrm{D}$ TDSE,

$$
i \frac{\partial}{\partial t} \Psi(\vec{r}, t)=\left[-\frac{1}{2} \nabla^{2}+V_{\mathrm{eff}}(r)-\vec{A}(t) \cdot \vec{p}\right] \Psi(\vec{r}, t) .
$$

This equation describes the spatiotemporal evolution of the electronic wave function experiencing an effective, spherically symmetric, one-electron molecular potential $V_{\text {eff }}(r)$ and interacting with the laser field within the dipole approximation (expressed in the velocity gauge via the term $\vec{A} \cdot \vec{p}$ ). A $B$-spline method is used to solve Eq. (1) [17]. The momentum distribution of the emitted electrons is calculated by projecting the final electronic wave function onto an 
incoming continuum wave function.

$$
\frac{\partial^{2} P}{\partial k \partial \theta_{k}}=\int\left|\left\langle f_{\vec{k}}^{(-)} \mid \Psi(t=+\infty)\right\rangle\right|^{2} d \varphi_{k},
$$

with

$$
\begin{aligned}
\left\langle\vec{r} \mid f_{k}^{(-)}\right\rangle & \equiv f_{\vec{k}}^{(-)}(\vec{r}) \\
& =\sum_{l, m} i^{l} e^{-i \Delta_{l}} \phi_{k}^{l}(r) \mathrm{Y}_{l, m}\left(\theta_{k}, \varphi_{k}\right) \mathrm{Y}_{l, m}^{*}\left(\theta_{k}, \varphi_{k}\right),
\end{aligned}
$$

where $l$ and $m$ denote, respectively, the angular and magnetic quantum numbers of the partial waves, $\phi_{k}^{l}(r)$ is the radial wave function of momentum $k$, and $\mathrm{Y}_{l, m}^{*}\left(\theta_{k}, \varphi_{k}\right)$ are the spherical harmonics. The phase shift $\Delta_{l}=\eta_{l}+\delta_{l}$ is the sum of the Coulomb phase $\eta_{l}=\arg \Gamma\left(l+1-i \frac{z}{k}\right)$ with $z=1$ the asymptotic charge seen by the ejected electron and $\delta_{l}$, a short-range scattering phase shift. The procedure used here is explained in full detail in Ref. [18].

The molecular potential $V_{\text {eff }}(r)$ appearing in Eq. (1) was obtained by a local density approximation (LDA) calculation within the framework of density functional theory (DFT) and the spherical jellium model. In the Kohn-Sham formulation of DFT [19], the ground-state electron density $n_{e}(\vec{r})$ of an $N$-electron system is written, in terms of single-particle orbitals $\psi_{k}$ and energies $\varepsilon_{k}$, as

$$
n_{e}(\vec{r})=\sum_{k=1}^{\text {occ }}\left|\psi_{k}(\vec{r})\right|^{2},
$$

where $o c c$ is the number of occupied orbitals. These orbitals and energies obey the Schrödinger equation:

$$
\left[-\frac{1}{2} \nabla^{2}+V_{\mathrm{eff}}(r)\right] \psi_{i}(\vec{r})=\varepsilon_{i} \psi_{i}(\vec{r})
$$

where $V_{\text {eff }}(r)$ is an effective single-particle potential which is found in an iterative way following the usual DFT approach. The effective potential is given by $V_{\text {eff }}(r)=V_{\text {ext }}(r)+V_{H}(r)+$ $V_{x c}(r)$, where $V_{\text {ext }}(r)$ is an external potential (e.g., due to the ionic background), $V_{H}(r)$ is the Hartree potential solution of Poisson's equation, and $V_{x c}(r)$ is the exchangecorrelation potential. Following Perdew and Zunger [20] we have added a self-interaction correction that restores the correct asymptotic behavior of the effective potential. $V_{\text {ext }}$ was treated in the spherical jellium approximation following the model developed by Bauer et al. [21]. In the latter, the charge of the real ionic cores is replaced by a constant positive background uniformly distributed over a spherical shell. Previous studies have demonstrated [22] that this model is able to mimic the features that are obtained by using much more sophisticated first-principle calculations [23]. The electronic states are labeled by the $\left(l, n_{r}, m\right)$ quantum states standing for the orbital angular momentum, the radial, and the magnetic quantum number. $n_{r}$ is equal to the number of nodes in the radial wave function [for the $\sigma(\pi)$ states $n_{r}=0(1)$ ]. The analysis of the electron momentum distribution and the average procedure over the focal volume (needed to compare with the experimental measurements) being very numerically demanding we have chosen to use this rather simple modeling of the electronic structure.

The statistical model that we use [24] was successfully applied to thermoelectronic emission from highly excited sodium clusters. In the following we briefly describe how this approach is adapted to $\mathrm{C}_{60}$ molecules irradiated by an intense and short laser pulse. The kinetic energy distribution of electrons emitted from $\mathrm{C}_{60}^{i+}(i=0,1, \ldots)$ species is given by

$$
P_{i}(\varepsilon) \propto \int_{t=0}^{\propto} N_{i}(t) k_{i}\left(E_{i}(t), \varepsilon\right) d t,
$$

where $\varepsilon$ is the electron kinetic energy, $N_{i}(t)$ is the population of $\mathrm{C}_{60}^{i+}$ at time $t$ determined by solving a system of coupled firstorder differential equations, and $k_{i}\left(E_{i}(t), \varepsilon\right)$ is the differential electron emission rate for the production of electrons with a kinetic energy $\varepsilon$ from $\mathrm{C}_{60}^{i+}$ ions with a time-dependent internal energy $E_{i}(t)$, and is evaluated within the framework of the statistical model proposed by Weisskopf [25],

$$
k_{i}\left(E_{i}(t), \varepsilon\right)=\frac{2}{\pi^{2}} \sigma_{i}(\varepsilon) \varepsilon \frac{\rho\left(E_{i}(t)-V_{\text {ion }}^{i}-\varepsilon\right)}{\rho\left(E_{i}(t)\right)},
$$

where $\sigma_{i}(\varepsilon)$ is the classical capture cross section of a Coulomb plus hard sphere interaction potential, $\rho\left(E_{i}(t)\right)$ the electronic level density which is approximated by that of an ideal Fermi gas, and $E_{i}(t)$ the internal energy of $\mathrm{C}_{60}^{i+}$ which is evaluated by assuming that the electron gas is heated up by photoabsorption and cooled down by energy transfer to the ionic background (the phonons). The values of the ionization potentials, $V_{\text {ion }}^{i}$, have been taken from Ref. [26] and have been obtained from quantum chemistry calculations using DFT with the B3LYP functional for exchange and correlation. It is also mentioned in [26] that the ionization potentials may be well reproduced by using the simple formula $V_{\text {ion }}^{i}[\mathrm{eV}]=7.108+3.252 i$.

We first discuss the result of the TDSE calculation. Calculations of the ionization rates show that for $I=2 \times 10^{13} \mathrm{~W} / \mathrm{cm}^{2}$ the HOMO $\left(l=5, n_{r}=1, m\right)$ state dominates the ionization yield by several orders of magnitude over the HOMO-1 $\left(l=4, n_{r}=1, m\right)$ orbital. Therefore, we will only consider ionization of this most probable channel. Figure 2(d) shows the computed ionization rate of various $|m|$ components of the $l=5$ state as a function of the laser intensity. Interestingly, the ionization efficiency is very similar for $|m|=0$ and 1 . Because of the twofold $m$-degeneracy, the largest contribution to the ionization rate is therefore given by the $|m|=1$ states rather than $m=0$. This is a direct consequence of the fact that the $\mathrm{C}_{60}$ HOMOs are high angular momentum states for which the wave function corresponding to the lowest projection of $l$ (i.e., $|m| \ll l)$ are very similar. Therefore, these states have nearly identical light-induced dipoles and display very similar ionization behavior, in contrast to the usual atomic situation, where the ground state has a low angular momentum state and $m=0$ often dominates. Note also that contrary to the atomic case the electronic structure of $\mathrm{C}_{60}$ is extremely dense and the plethora of excited states reached via multiphoton transitions prevents the appearance of given isolated resonances in the ATI electron spectrum.

Let us now discuss the angular distributions. In Fig. 2(a), a calculated two-dimensional (2D) momentum distribution over a large electron kinetic energy range is presented for a laser intensity of $2 \times 10^{13} \mathrm{~W} / \mathrm{cm}^{2}$ ionizing the $m=0$ state. This momentum distribution is peaked along the polarization axis, and contains a series of side lobes. Similar patterns were recently observed in strong-field ionization of metastable 
(a)

(d)
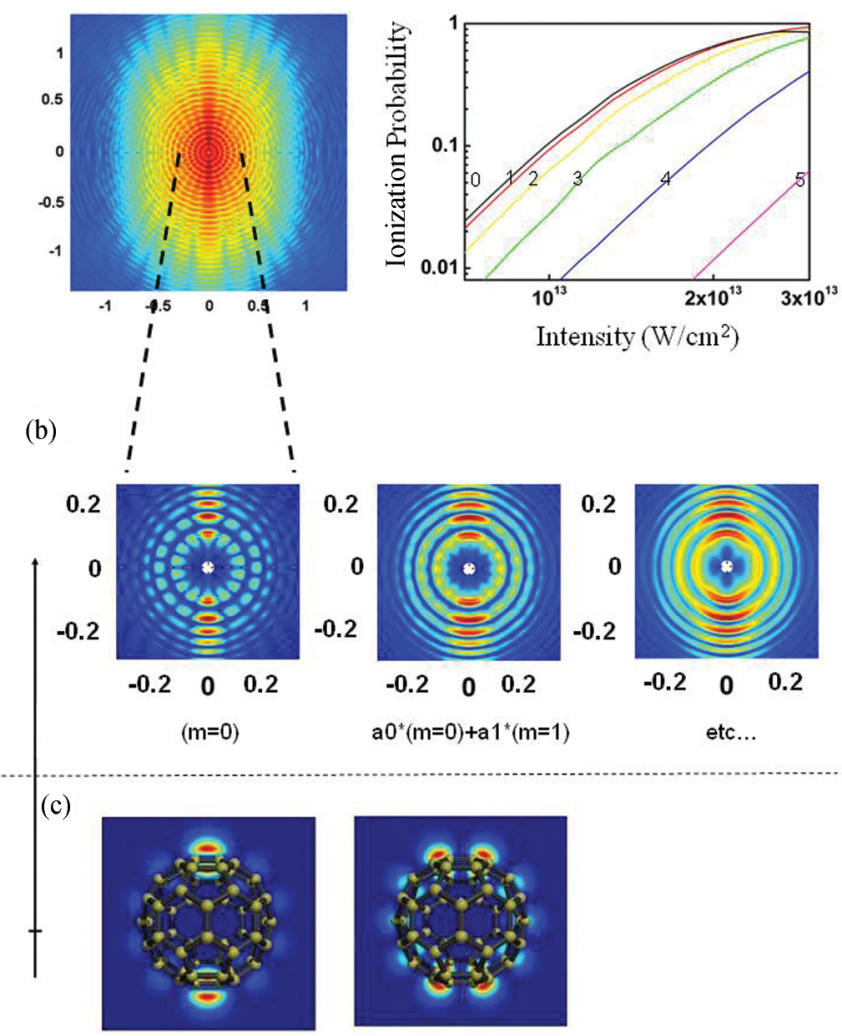

FIG. 2. (Color online) (a) TDSE calculation for $I=2 \times$ $10^{13} \mathrm{~W} / \mathrm{cm}^{2}$ of the momentum distribution; side lobes are observed that could correspond to the interference between electron wave packets emitted within a single quarter cycle of the light pulse. (b) The electron momentum distributions for the individual $\left(l=5, n_{r}=1, m\right)$ $\pi$ states, where we have successively added the contribution of all $m$ states. For instance, the second figure corresponds to the sum of the contribution of the orbital $m=0$ and $m=1$ weighted by their respective ionization yield $a 0$ and $a 1$. The last depicted picture shows the result after adding all $m$-state contributions. In the total momentum signal the nodal pattern completely disappeared leading to a smooth, structureless aligned angular distribution in agreement with the experimental results. (c) Calculated individual $\pi$ states $(l=5$, $\left.n_{r}=1, m=0\right)$ and $\left(l=5, n_{r}=1, m=1\right)$. (d) Calculated ionization rate for $\left(l=5, n_{r}=1, m=0 \cdots 5\right)$ states showing the importance of the first $m$ states.

Xe atoms at mid-IR wavelengths [3], and interpreted in terms of a holographic interference between electrons that leave the atom without a strong interaction with the ion left behind and electrons that recollide with their parent ion. The lobes that we observe persist in a single laser cycle calculation and might originate from this holographic process. In such mechanism, the electron wave function is split into two parts; the first part corresponds to an electron that is directly ejected into the continuum and barely affected by the ionic core, while the second part of the wave function is ejected, accelerated back to the ion by the light electric field. This part of the wave scatters on the core and interferes with the first part of the wave function. The interference between the scattered and reference electronic wave leads to a holographic pattern. As in optics, it carries information about the object which diffracted the incident wave (in our case the molecular potential).

Let us now concentrate on the low electron kinetic energy part of the spectrum. In Fig. 2(b), we present the calculated 2D photoelectron momentum distributions resulting from ionization by a $2 \times 10^{13} \mathrm{~W} / \mathrm{cm}^{2}$ laser field of the $\left(l=5, n_{r}=\right.$ $1, m)$ initial orbitals. Some examples of the corresponding ionized $\left(l=5, n_{r}=1, m\right)$ orbitals are represented in Fig. 2(c). The 2D momentum distribution is highly structured for each $m$ but the angular distributions for the different $m$-states are different from each other. As a result the incoherent addition of the first $2 \mathrm{~m}$-states taking into account the relative ionization rate already leads to an almost structureless angular distribution [Fig. 2(b)]. The momentum distribution summed over all the $m$-projections is very smooth and becomes narrower for the high-order ATI peaks, in very good agreement with the experiment. We conclude that the remarkably simple angular distributions observed in the strong-field ionization of $\mathrm{C}_{60}$ can be modeled as atomiclike SAE ionization processes. They are caused by the accumulation of highly structured but slightly different angular distributions from the various $m$-components of a single high angular momentum electronic ground state. Although they could also play a role in the final angular distribution, no other effect related to the complexity of the molecule needs to be invoked.

However, the angular distribution is only a part of the information given by the VMIS measurements. In fact that SAE calculation does not fully describe these measurements shown in Fig. 1. This becomes readily apparent when we compare the measured angle-integrated photoelectron spectrum and the SAE calculation in Fig. 3. While the position of the ATI peaks and their relative amplitude is relatively well reproduced by the TDSE calculation, a large difference in the peak-to-valley contrast is observed. As previously shown by Campbell et al. [7,27], this is not a consequence of

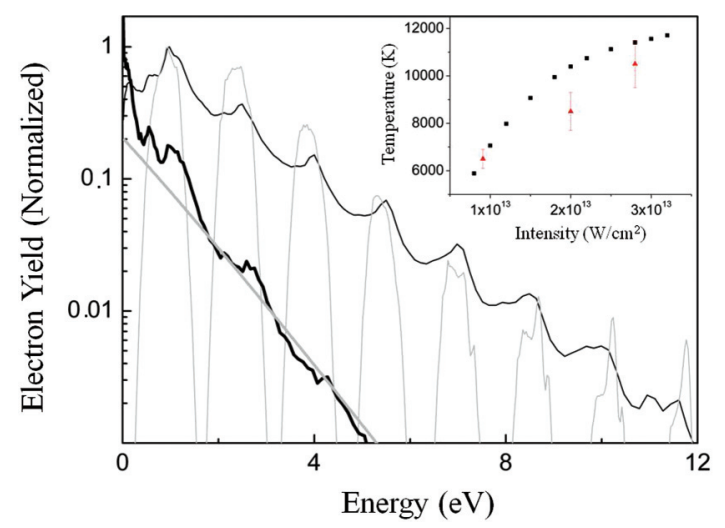

FIG. 3. (Color online) Comparison between experimental and calculated electron spectra. The TDSE calculation predicts a high contrast of the ATI peaks (thin gray curve). The loss of contrast observed in the experimental data (thin black curve) is attributed to electron-electron interaction. A thermal calculation (solid gray curve) leads to fair agreement with the experimental background measured at $90^{\circ}$ with respect to the laser polarization (solid black curve). Inset: Comparison between calculated temperature (black squares) and temperature extracted at $90^{\circ}$ from the velocity distribution (red triangles) is shown. 
experimental resolution but it characterizes a real presence of an isotropic electron distribution in the images. The origin of this background lies in a thermoelectronic emission process, where the ionization occurs from a hot electron gas. It is known that this process becomes dominant when the pulse duration reaches a few hundred femtoseconds and signifies the importance of relaxation processes through electron-electron interactions. Statistical theories for finite-size systems can be used to describe this mechanism as presented in the preceding theory section [28]. The calculated thermal contribution to the photoelectron spectrum shows a smooth, near exponential-like shape from which an apparent temperature can be derived. In order to disentangle the ATI and thermal emission, we use the fact that the ATI process is mostly aligned along the laser polarization axis while the purely thermal emission is isotropic. Therefore, the thermal contribution can be obtained by considering the photoemission that occurs at $90^{\circ}$ with respect to the laser polarization where the ATI contribution is expected to be minimal. Experimental temperatures were then obtained by a fitting procedure, which can be compared to theoretical values following from the statistical model. This comparison is shown in the inset of Fig. 3 for several laser intensities. The agreement is good. In Fig 3 the experimental photoelectron spectra recorded at $2 \times 10^{13} \mathrm{~W} / \mathrm{cm}^{2}$ and the $90^{\circ}$ contribution are compared to, respectively, the focal volume averaged TDSE and the statistical model. The major difference that remains between the experiment and our calculations originates from the effect of the light electric field on the thermal contribution [29]. The light electric field induces an increase of the electron kinetic energy along the laser polarization which considerably increases the apparent temperature along this axis. Using angle-resolved measurements at $90^{\circ}$ allows an accurate determination of the effective thermal contribution by disentangling this effect.

In conclusion, we have measured the velocity distribution of photoelectrons emitted from $\mathrm{C}_{60}$ molecules irradiated by an intense short IR femtosecond laser pulse. TDSE calculations starting from individual molecular states show a very structured angular distribution assigned to the high angular momentum of the continuum wave function and to holographic effects. By contrast, the experimental angular distribution of the ATI peaks is structureless. As demonstrated, understanding this effect does not require to invoke the complexity of the molecule. On the contrary, $\mathrm{C}_{60}$ behaves as a "macro-atom" for which single-electron ionization from a one-electron orbital produces the observed effect. Unlike the usual atomic situation, the $|m|=1$ states dominate the ionization and the contribution of all $m$-states leads to a smooth angular distribution. Beyond this atomiclike effect, we have identified a loss of contrast in the ATI peaks that cannot be reproduced by SAE processes. This effect, already pointed out in the literature, is related to the influence of electron-electron interactions and can be satisfactorily calculated using a statistical approach if the focal volume averaging is taken into account. This demonstrates that important information on the ionization dynamics can be obtained in ATI measurements for complex systems. Our work has allowed disentangling the various mechanisms at play in the $\mathrm{C}_{60}$ ionization while a fully satisfactory theoretical description would require a complete $a b$ initio calculation that would encounter for both coherent and thermal-like ionization mechanisms. Such calculation is compulsory for the global description of complex molecular systems interacting with a laser field.

We acknowledge the Huber Curien program Van Gogh 13800UG, Grant No. GDR 2758 “Thermodynamique, Fragmentation et Agrégation de systèmes moléculaires complexes isolés," and Grant No. ANR-10-BLAN-0428-01 for financial support. This work is part of the research program of the "Stichting voor Fundamenteel Onderzoek der Materie" (FOM), which is financially supported by the "Nederlandse Organisatie voor Wetenschappelijk Onderzoek" (NWO). We thank Khadga Karki for his valuable contribution during the experimental run.
[1] R. Kienberger, R. Kienberger, M. Hentschel, M. Uiberacker, Ch. Spielmann, M. Kitzler, A. Scrinzi, M. Wieland, Th. Westerwalbesloh, U. Kleineberg, U. Heinzmann, M. Drescher, and F. Krausz, Science 297, 1144 (2002).

[2] W. Becker, F. Grasbon, R. Kopold, D. B. Milosevic, G. G. Paulus, and H. Walther, Adv. At. Mol. Opt. Phys. 48, 35 (2002).

[3] R. R. Freeman, P. H. Bucksbaum, H. Milchberg, S. Darack, D. Schumacher, and M. E. Geusic, Phys. Rev. Lett. 59, 1092 (1987).

[4] Y. Huismans, A. Rouzée, A. Gijsbertsen, J. H. Jungmann, A. S. Smolkowska, P. S. W. M. Logman, F. Lépine, C. Cauchy, S. Zamith, T. Marchenko, J. M. Bakker, G. Berden, B. Redlich, A. F. G. van der Meer, H. G. Muller, W. Vermin, K. J. Schafer, M. Spanner, M. Yu. Ivanov, O. Smirnova et al., Science 331, 61 (2010).

[5] E. V. van der Zwan and M. Lein, Phys. Rev. Lett. 108, 043004 (2012).
[6] M. Meckel, D. Comtois, D. Zeidler, A. Staudte, D. Pavičić, H. C. Bandulet, H. Pépin, J. C. Kieffer, R. Dörner, D. M. Villeneuve, and P. B. Corkum, Science 320, 1478 (2008).

[7] F. Rohmund, M. Heden, A. V. Bulgakov, and E. E. B. Campbell, J. Chem. Phys. 115, 3068 (2001).

[8] E. E. B. Campbell, K. Hansen, K. Hoffmann, G. Korn, M. Tchaplyguine, M. Wittmann, and I. V. Hertel, Phys. Rev. Lett. 84, 2128 (2000).

[9] F. Lépine and C. Bordas, Phys. Rev. A 69, 053201 (2004).

[10] I. Shchatsinin, T. Laarmann, G. Stibenz, G. Steinmeyer, A. Stalmashonak, N. Zhavoronkov, C. P. Schulz, and I. V. Hertel, J. Chem. Phys. 125, 194320 (2006).

[11] M. Boyle, T. Laarmann, K. Hoffmann, M. Heden, E. E. B. Campbell, C. P. Schulz, and I. V. Hertel, Eur. Phys. J. D 36, 339 (2005).

[12] I. V. Hertel, I. Shchatsinin, T. Laarmann, N. Zhavoronkov, H.-H. Ritze, and C. P. Schulz, Phys. Rev. Lett. 102, 023003 (2009). 
[13] A. Jaroń-Becker, A. Becker, and F. H. M. Faisal, Phys. Rev. Lett. 96, 143006 (2006).

[14] M. Ruggenthaler, S. V. Popruzhenko, and D. Bauer, Phys. Rev. A 78, 033413 (2008).

[15] A. T. J. B. Eppink and D. H. Parker, Rev. Sci. Instrum. 68, 3477 (1997).

[16] G. G. Paulus, F. Grasbon, A. Dreischuh, H. Walther, R. Kopold, and W. Becker, Phys. Rev. Lett. 84, 3791 (2000).

[17] H. Bachau, E. Cormier, P. Decleva, J. E. Hansen, and F. Martin, Rep. Prog. Phys. 64, 1815 (2001).

[18] E. Cormier, D. Garzella, P. Breger, P. Agostini, G. Chériaux, and C. Leblanc, J. Phys. B 34, L9 (2001).

[19] W. Kohn and L. J. Sham, Phys. Rev. 140, A1133 (1965).

[20] J. P. Perdew and A. Zunger, Phys. Rev. B 23, 5048 (1981).

[21] D. Bauer, F. Ceccherini, A. Macchi, and F. Cornolti, Phys. Rev. A 64, 063203 (2001).

[22] M. J. Puska and R. M. Nieminen, Phys. Rev. A 47, 1181 (1993); M. Boyle, K. Hoffmann, C. P. Schulz, I. V. Hertel, R. D. Levine, and E. E. B. Campbell, Phys. Rev. Lett. 87, 273401 (2001); L. F. Ruiz, P.-A. Hervieux, J. Hanssen, M. F. Politis, and F. Martin, Int. J. Quantum Chem. 86, 106 (2002); A. Rüdel, R. Hentges, U. Becker, H. S. Chakraborty, M. E. Madjet, and J. M. Rost, Phys. Rev. Lett. 89, 125503 (2002); E. Cormier,
P.-A. Hervieux, R. Wiehle, B. Witzel, and H. Helm, Eur. Phys. J. D 26, 83 (2003); A. Rentenier, L. F. Ruiz, S. Díaz-Tendero, B. Zarour, P. Moretto-Capelle, D. Bordenave-Montesquieu, A. Bordenave-Montesquieu, P.-A. Hervieux, M. Alcamí, M. F. Politis, J. Hanssen, and F. Martín, Phys. Rev. Lett. 100, 183401 (2008); Y. Pavlyukh and J. Berakdar, Chem. Phys. Lett. 468, 313 (2009).

[23] G. F. Bertsch, A. Bulgac, D. Tomanek, and Y. Wang, Phys. Rev. Lett. 67, 2690 (1991); N. Troullier and José Luis Martins, Phys. Rev. B 46, 1754 (1992).

[24] R. Schlipper, R. Kusche, B. von Issendorff, and H. Haberland, Appl. Phys. A 72, 255 (2001).

[25] V. Weisskopf, Phys. Rev. 52, 295 (1937).

[26] S. Díaz-Tendero, M. Alcamí, and F. Martín, J. Chem. Phys. 123, 184306 (2005).

[27] M. Kjellberg, O. Johansson, F. Jonsson, A. V. Bulgakov, C. Bordas, E. E. B. Campbell, and K. Hansen, Phys. Rev. A 81, 023202 (2010).

[28] K. Hansen, K. Hoffmann, and E. E. B. Campbell, J. Chem. Phys. 119, 2513 (2003).

[29] J. O. Johansson, J. Fedor, M. Goto, M. Kjellberg, J. Stenfalk, G. G. Henderson, E. E. B. Campbell, and K. Hansen, J. Chem. Phys. 136, 164301 (2012). 